\title{
Proxy System modeling and data assimilation in paleosciences
}

\author{
Mike Evans', H. Goosse ${ }^{2}$ and S. Khatiwala ${ }^{3}$ \\ Louvain-la-Neuve, Belgium, 29 May - 1 June 2017
}

Data assimilation (DA) for paleoenvironmental reconstruction combines information from observations and models of the Earth system to develop mechanistically consistent estimates of environmental fields. There are four essential ingredients to paleoenvironmental DA: (1) Models of the Earth system describing possible system states; (2) Observations telling us what actually happened in the real world; (3) Proxy System Models (PSMs) describing how geological, biological or chemical archives are imprinted with environmental signals, and linking process models to observations; and (4) An algorithm that optimally combines model results, observations, and uncertainties in the various elements.

To review current activities in paleoenvironmental DA, a group of climate scientists recently met in Louvain-la-Neuve, Belgium. The group contained specialists of climate modeling and scientists more closely involved in the collection and analysis of paleoclimate observations. The goal was to consider challenges and potential for advances in DA and PSMs, and to stimulate new activities via practical training exercises.

\section{Challenges and opportunities}

Overview talks first reviewed the activities of a small but rapidly growing community. The parallels with modern DA were particularly inspiring. In addition to the product itself, reanalyzes are an opportunity to bring together scientists of different backgrounds, stimulate data recovery, and demonstrate skill improvement brought by additional data.

The talks and subsequent breakout groups identified many existing PSMs of varying structure and complexity for a number of major paleoenvironmental archives and observations. However, the uncertainty in PSMs - structural and/or systematic - is not well constrained. There are also multiple DA techniques that might be applied to paleoenvironmental reconstructions but no assessment of their relative advantages and limitations in settings corresponding to paleoclimate applications is currently available. Therefore, the choice of PSM for a particular DA problem is frequently unclear.

The workshop concluded with four classroom exercises introducing participants to proxy system modeling, adjoint methods and data assimilation.

\section{Recommendations of the workshop}

The participants proposed three activities to be launched in the coming months:

(1) Use case studies for particular replicated observations, proxy systems and archives to better describe the structural uncertainty and systematic error in PSMs and in observations as it seems important to first test the PSM independently of Earth system models.

(2) Create a testbed for the development and testing of paleo DA methods constrained by a realistic observing network

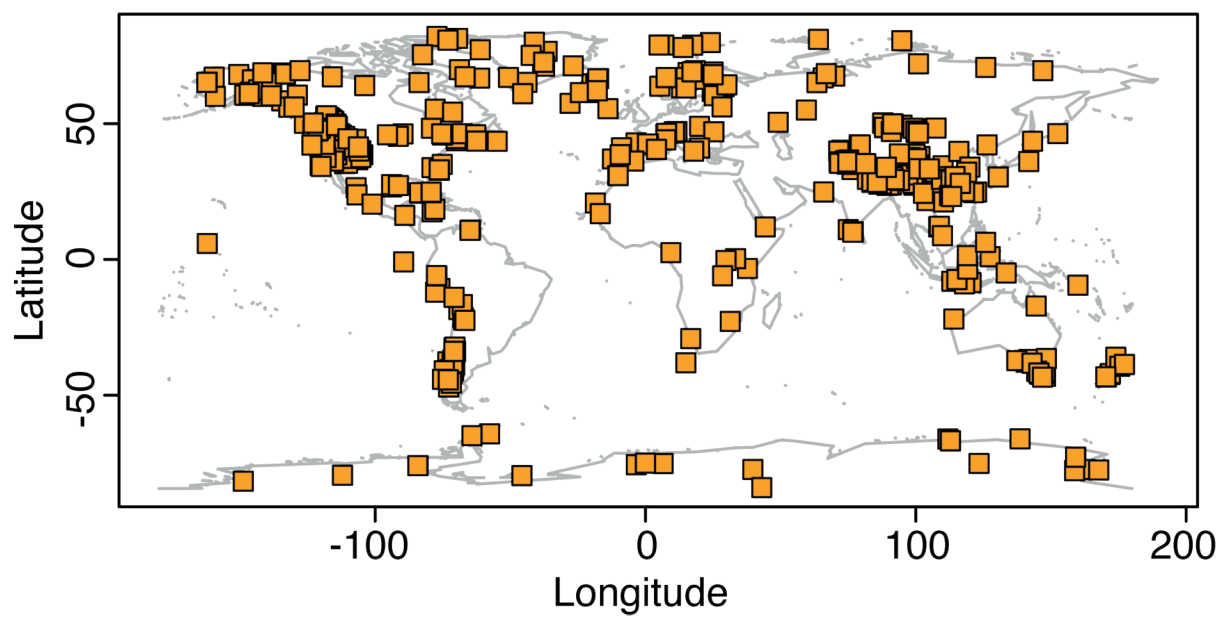

corresponding to the past millennium, but using data for the last century with specific additional noise (Fig. 1), and compare the reconstructions to modern reanalysis products.

(3) Form a task force to explore uncertainty quantification procedures relevant to paleoclimate reconstructions, develop metrics to assess their relative merits, and to apply these to parameter estimation and data assimilation problems.

As a first step, the proposed activities are thus either devoted to the modeling of a specific type of record or to the comparison of DA methods in an idealized framework where observations are modeled variables with a precisely known uncertainty. First results of these activities are expected in early 2018 so that they can be presented at a planned DAPS session at EGU. As the ultimate goal of DAPS is to bridge the gap between modeling of a wide range of data and data assimilation, the next DAPS workshop in the second half of 2018 will be devoted to issues that limit a global approach and the means to tackle them. The path to solve those problems will be put forward in 2019.

DAPS is an open community of observationalists and modelers, and we invite you to bring your interests and expertise into the project. For more information, visit the DAPS website (http://pastglobalchanges.org/ ini/wg/daps/intro) where the experimental design of the planned intercomparisons will be posted, join the working group mailing list (https://listserv.unibe.ch/mailman/listinfo/daps.pages) or email one of the coordinators.

A copy of the talks presented at the workshop is available here: http://pastglobalchanges.org/calendar/2017/127-pages/1657daps-1st-wshop-2017

\section{AFFILIATIONS}

'Department of Geology, University of Maryland, College Park, USA

2Earth and Life Institute, Université catholique de Louvain, Belgium

${ }^{3}$ Department of Earth Sciences, University of Oxford, UK

CONTACT

Mike Evans: mnevans@umd.edu

REFERENCES

PAGES 2k Consortium (2017) Sci data 4: 170088

Figure 1: Example of the data distribution for the comparison of data assimilation methods, derived from the data distribution in 1500 CE in the PAGES 2k database (PAGES 2k Consortium 2017). Figure courtesy of J. Franke. 